\title{
A $\alpha$-glycerophosphate Dehydrogenase is Present in Trypanosoma cruzi Glycosomes
}

\author{
JL Concepcion, H Acosta, W Quiñones, M Dubourdieu+
}

\author{
Unidad de Bioquímica de Parásitos, Centro de Ingenería Genética, Facultad de Ciencias, \\ Universidad de Los Andes, Apartado 38, Mérida, Venezuela
}

$\alpha$-glycerophosphate dehydrogenase ( $\alpha$-GPDH-EC.1.1.1.8) has been considered absent in Trypanosoma cruzi in contradiction with all other studied trypanosomatids. After observing that the sole malate dehydrogenase can not maintain the intraglycosomal redox balance, GPDH activity was looked for and found, although in very variable levels, in epimastigotes extracts. GPDH was shown to be exclusively located in the glycosome of $\mathrm{T}$. cruzi by digitonin treatment and isopycnic centrifugation. Antibody against $\mathrm{T}$. brucei GPDH showed that this enzyme seemed to be present in an essentially inactive form at the beginning of the epimastigotes growth. GPDH is apparently linked to a salicylhydroxmic-sensitive glycerophosphate reoxidizing system and plays an essential role in the glycosome redox balance.

Key words: $\alpha$-glycerophosphate dehydrogenase - glycosome - Trypanosoma cruzi

Trypanosoma cruzi, like all known trypanosomatids, presents an unusual glucose metabolism. First, the 7-8 initial enzymes of the glycolytic pathway are compartmentalized in a microbody-type organelle, the glycosome (Opperdoes 1987, Taylor \& Guttridge 1987). Second, the oxidative degradation of glucose is not complete: only part of the glucose carbon appears as $\mathrm{CO}_{2}$ and the rest is excreted as reduced organic compounds, mainly succinate and alanine in $T$. cruzi epimastigotes (Cazzulo 1994). The incomplete breakdown of glucose into succinate is related to the presence in the glycosome of an auxiliary system, constituted by an ATP-producing phosphoenolpyruvate carboxykinase (PEPCK) and a malate dehydrogenase (MDH) (Urbina 1994), which transforms phosphoenolpyruvate (PEP) to malate by fixing $\mathrm{CO}_{2}$ and oxidizing reduced nicotinamide dinucleotide (NADH). Malate is eventually converted to succinate in the mitochondrion. This glycosomal system is present in all stages of the life cycle of T. cruzi (Urbina et al. 1993). Theoretically, $\mathrm{MDH}$ is able to reoxidize all the glycosomal NADH produced by glycolytic glyceraldehydephosphate dehydrogenase. Practi-

This work was supported by CDCHT-ULA to MD (grant C-630).

${ }^{+}$Corresponding author. Fax: 58-74-401286. E-mail: dubourdi@ciens.ula.ve

Received 1 August 2000

Accepted 5 February 2001 cally, for that to be so, all the PEP (2 moles per glucose) produced in the cytosol as a consequence of the glycosomal glycolytic activity, should reenter the glycosome; and that is not possible due to the presence of a low cytosolic pyruvate kinase activity which converts part of the PEP to pyruvate. If the glycosomal membrane is considered as a true permeability barrier towards molecules such as oxidizing reduced nicotinamide dinucleotide (NADH), the existence of at least another glycosomal $\mathrm{NAD}^{+}$-regenerating system, besides $\mathrm{MDH}$, has to be postulated in order to account for the glycosome redox balance. The other dehydrogenase known to play a role in the redox balance of the glycosome of T. brucei, $\alpha$-glycerophosphate dehydrogenase (GPDH), has not been detected in T. cruzi (Taylor \& Gutteridge 1987, Cazzulo et al. 1988) although it is present in all the other known trypanosomatidae (Tielens \& Van Hellemond 1998). In these organisms, GPDH is part of a system which enables the reoxidation of glycosomal NADH by molecular oxygen through the classical dihydroxyacetone-phosphate/ $\alpha$-glycerophosphate shuttle and a mitochondrial alternative $\alpha$-glycerophosphate oxidase or GPO (Tielens \& Van Hellemond 1998). It is therefore noteworthy that different authors have observed an inhibition of oxygen consumption by salicylhydroxamic acid (SHAM) in T. cruzi epimastigotes (Felix et al. 1978, Stoppani et al. 1980, Engel et al. 1990), and even the direct oxidation of glycerophosphate by mitochondrial extracts (Affranchino et al. 1985), an indication of the possible presence of a GPO system (generally refered as SHAM-sensitive alternative oxidase) and also of a $\alpha$-glycerophosphate generat- 
ing system. We therefore decided to have another look at $T$. cruzi GPDH (EC 1.1.1.8) activity.

Epimastigotes (EP strain) were cultivated, as already described by Concepcion et al. (1999), in LIT medium supplemented with $10 \mathrm{mM}$ glucose and harvested at mid-exponential phase (around $0.6 \mathrm{OD}$ at $600 \mathrm{~nm}$ ) or as pointed out in the following experiments: (1) GPDH activity was determined on whole epimastigote extracts in both directions in order to avoid misinterpretations due to other NAD+/NADH-utilizing systems (Niesel et al. 1982). Only one extract out of nine (corresponding to independent cultures) did not present any detectable activity. In the other extracts GPDH specific activity varied from about $0.3 \mathrm{U} / \mathrm{mg}$ protein (two cases) down to $0.005 \mathrm{U} / \mathrm{mg}$ protein depending of the culture, with a mean value of about 0.07 to be compared with 0.75 and $0.15 \mathrm{U} / \mathrm{mg}$ protein for phosphoglucose isomerase (PGI) and hexokinase (HK) respectively. Fig. 1A shows that GPDH specific activity, which was not detectable at the beginning of the exponential phase, increased during growth. However an antiserum raised against $T$. brucei GPDH evidenced a maximum of cross-reacting protein at the beginning of the growth. Put together, these two observations indicated that most of the enzyme expressed during early exponential phase was inactive (Fig. 1B); (2) $T$. cruzi epimastigotes were permeabilized with increasing amounts of digitonin as already described by Concepcion et al. (1999). GPDH was liberated at the same digitonin concentrations as for HK, the classical glycosomal marker (Fig. 2), in agreement with a glycosomal localization. Phosphoglucose isomerase was liberated in a biphasic manner as expected for an enzyme presenting a dual location (Concepcion et al. 1999); (3) glycosomes were purified by differential and isopycnic centrifugations according to Concepcion et al. (1998, 1999). GPDH activity distribution was absolutely identical to those of PEPCK and HK by differential (not shown, see Concepcion et al. 1998) and isopycnic centrifugations (Fig. 3). The western blot performed in the presence of the antiGPDH antibody exhibited the same distribution as the enzymatic activity (not shown), indicating an essentially exclusive glycosomal location of GPDH. In this experiment, the purified glycosomes presented latencies of $92 \%$ for GPDH, $87 \%$ for HK and $80 \%$ for PGI; (4) an estimation of GPO activity was obtained by determining SHAM inhibition of the oxygen consumption rate of whole cells in a Warburg respirometer (each $3 \mathrm{ml}$ vial contained 1 $\mathrm{mg}$ of cell protein and $3 \mathrm{mM}$ glucose; the center well contained $0.3 \mathrm{ml}$ of $20 \%$ potassium hydroxide). The experiments were performed in the presence and absence of SHAM (1.2 mM) and/or cya- nide $(0.1 \mathrm{mM})$ on nine independently grown epimastigote cultures (harvested between 0.6 and 0.9 O.D. at $600 \mathrm{~nm}$ ). SHAM inhibited oxygen consumption by about $23 \%$ as an average but with variations between $0 \%$ (one case) and $57 \%$. Not only was the inhibitory effect of SHAM generally not additive with that of cyanide, but in two cases an antagonistic effect was observed. Antimycin inhibited between 90 and $100 \%$. Glucose consumption [measured as residual glucose according to Bergmeyer (1983)] was also inhibited by SHAM between 30 and $60 \%$, but not by cyanide. These results differed somewhat from those of Engel et al. (1990) who only observed SHAM inhibition on antimycin A inhibited cells and from those of Felix et al. (1978), but agree with them in indicating the likely presence of a GPO system in $T$. cruzi epimastigotes.

This work evidences that $T$. cruzi can synthesize, as do all other studied trypanosomatids (Tielens \& Van Hellemond 1998), a glycosomal

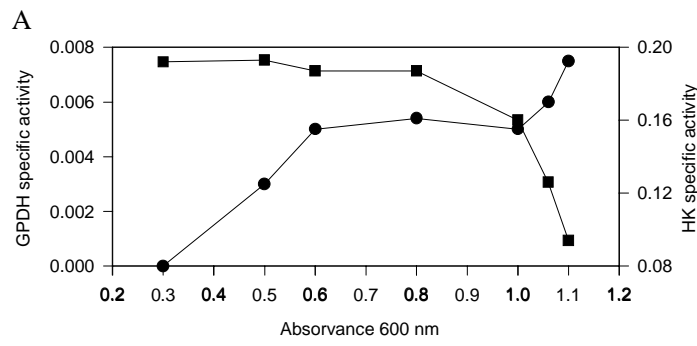

B

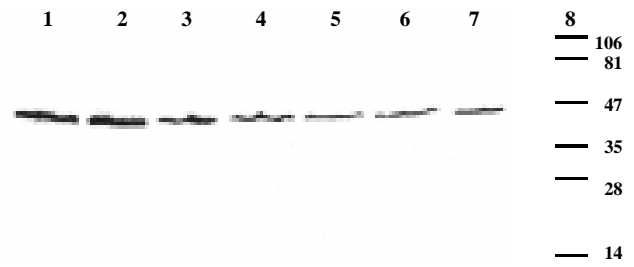

Fig. 1: glycerolphosphate dehydrogenase (GPDH) presence as a function of epimastigotes growth. Epimastigotes were grown as described previously (Concepcion et al. 1999) in a flask provided with a side-arm in order to follow the density of the culture (at $600 \mathrm{~nm}$ ). Cells samples were withdrawn at several times during growth, washed, treated with Triton $\mathrm{X}$ 100 and $0.15 \mathrm{M} \mathrm{NaCl}$ and analyzed for GPDH activity and GPDH protein by Western blot. A: GPDH (O) and hexokinase (HK) (ם) specific activities as a function of growth (absciss indicates the absorbance at harvesting time). Proteins and $\mathrm{HK}$ activity were determined as described previously by Concepcion et al. (1998). GPDH activity was determined according to Niesel et al. (1982); B: SDS-polyacrylamide gel electrophoresis of the cells extracts followed by Western blot was performed as described in Concepcion et al. (1999) in the presence of a rabbit antiserum raised against Trypanosoma brucei GPDH. Lanes 1-7 correspond to the 7 cell samples of A and lane 8 shows the molecular weight markers. Protein content was the same in all lanes. The observed band corresponded to an apparent molecular mass of $32 \mathrm{kDa}$. This experiment was realized twice with independently grown cells. 


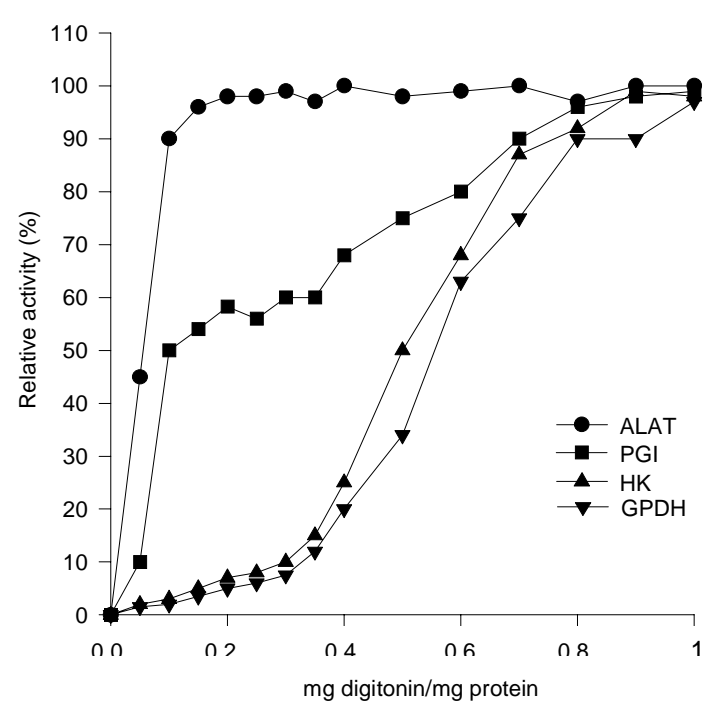

Fig. 2: digitonin treatment. The experiment was realized exactly as described by Concepcion et al. (1998, 1999); activities were determined in the supernatant of the centrifuged cells after $30 \mathrm{~min}$ incubation in the presence of the indicated proportion of digitonin to protein. The activities of alanine amino transferase (ALAT $\bullet$ ), phosphoglucose isomerase (PGI $\mathbf{\square}$ ), hexokinase (HK $\boldsymbol{\Delta}$ ) and glycerophosphate dehydrogenase (GPDH $\boldsymbol{\nabla}$ ) were determined according to Bergmeyer (1983). In the experiment presented, GPDH specific activity was 0.06 $\mathrm{U} / \mathrm{mg}$ of protein. This experiment was performed three times.

$\alpha$-glycerophosphate dehydrogenase. This should settle the contradictory issue raised by several independent reports in T. cruzi about the non-detectability of GPDH at the one hand (Taylor \& Guttridge 1978, Cazzulo et al. 1988), and the presence of a mitochondrial glycerolphosphate-oxidizing activity and a SHAM-sensitive oxidase at the other hand (Felix et al. 1978, Stoppani et al. 1980, Affranchino et al. 1985, Engel et al. 1990). The first observation is that, although the reason is not completely clear, GPDH and GPO activities were highly variable. Although the strain difference might explain differences in the expression levels of the oxidative systems (Engel et al. 1990), that seems unlikely since we have shown here that GPDH activity may or may not be detectable in the same strain grown under the same conditions. Harvesting time is evidently an essential factor: our results, but also those of Felix et al. (1978), show that GPDH is expressed differentially during epimastigotes growth, with a maximum in some moment of the late exponential phase. However, it is likely that essentially uncontrolled medium factors are also involved in this apparent incongruity. However the differential expression of the GPDH gene might not be the unique factor at stake. AntiGPDH antibody evidenced higher levels of the GPDH protein at the beginning of the growth curve than at the end, contrarily to what was observed with GPDH activity, thus indicating a possible posttranslational activation and a possible explanation for the variable levels of GPDH activity. The variability of GPDH expression/activity may be correlated to pyruvate kinase (PK) activity since more glycosomal GPDH would be necessary to maintain the redox balance in situations with relatively high PK activity and therefore less PEP re-entry in the glycosome. A 5-fold increase in PK specific activity was observed (unp. obs.) during late exponential phase of epimastigotes growth. In any case even low GPDH specific activities are compatible with an auxiliary role in maintaining the redox balance since PK activity is generally low. The glycerol production observed in $T$. cruzi amastigotes (Sanchez-Moreno et al. 1995) confirms the presence of a GPDH gene in T. cruzi. This gene is homologous to that of T. brucei as shown by the cross-reaction observed with an antiserum raised against $T$. brucei GPDH. The existence of at least two NADH-reoxidizing systems in the glycosome of $T$. cruzi gives possible explanations to several observations that could only be rationalized on the ground of a permeable glycosome membrane; for example, that the glycolytic activity was only little affected by a drastic inhibition of PEPCK (Urbina et al. 1990).

TABLE

Oxygen consumption of Trypanosoma cruzi epimastigotes in the presence of salicilic hydroxamate (SHAM) and cyanide $(\mathrm{CN})$

\begin{tabular}{|c|c|c|c|c|c|c|c|c|c|}
\hline SHAM (\%) & 94 & 60 & 57 & 43 & 82 & 92 & 83 & 100 & 82 \\
\hline $\mathrm{CN}^{-}(\%)$ & 26 & 13 & 17 & 56 & 28 & 25 & 47 & 50 & 49 \\
\hline $\mathrm{SHAM}+\mathrm{CN}^{-}(\%)$ & 78 & 0 & 28 & - & 23 & 49 & 51 & 51 & - \\
\hline
\end{tabular}

Each experiment was performed with independently grown cells harvested at mid-exponential phase (0.6 to 0.9 O.D. at $600 \mathrm{~nm}$ ). Oxygen consumption was determined with the Warburg respirometer at $28^{\circ} \mathrm{C}$ in the presence of 3 $\mathrm{mM}$ glucose after starving the cells in phosphate buffered-saline for about $4 \mathrm{~h} . \mathrm{CO}_{2}$ was trapped with $0.3 \mathrm{ml}$ of $20 \%$ potassium hydroxide in the center well of the flask. The results are expressed as the percentage of the oxygen consumption by the non-inhibited system (about $2 \mu$ moles/h). SHAM and CN concentrations were $1.2 \mathrm{mM}$ and 0.1 $\mathrm{mM}$ respectively. 
Hexokinase (96)

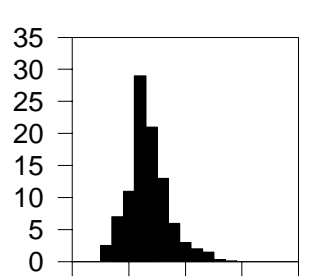

Glycerol-3-phosphate dehydrogenase (93)

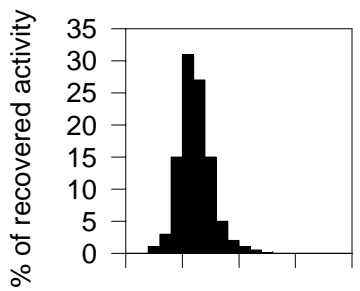

Phosphoenolpiruvate carboxikinase

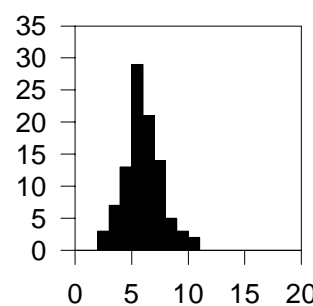

Isocitrate dehydrogenase (112)

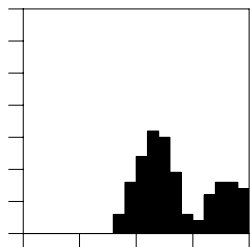

Succinate dehydrogenase $(98)$

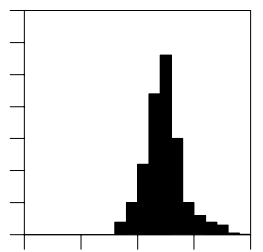

Density

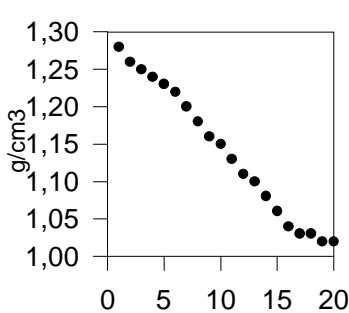

Cumulative volume
Alkaline

phosphatase (112)

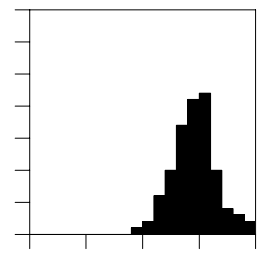

Esterase (108)

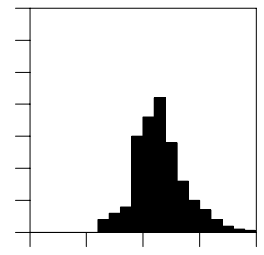

Protein (96)

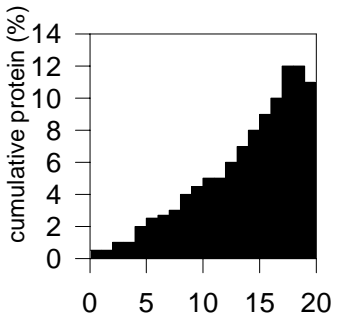

Fig. 3: glycerolphosphate dehydrogenase distribution profile after isopycnic centrifugation. A post-small granule fraction was run on a linear sucrose gradient $(0.3$ to $2.5 \mathrm{M})$ and 20 fractions were collected from the density gradient (density varied from 1.02 to $1.28 \mathrm{~g} . \mathrm{cm}^{-3}$ ). The experiment (repeated four times) was performed as described by Concepcion et al. (1998). The determination of enzymatic activities was performed according to Bergmeyer (1983) or as described in the previous experiments.

\section{ACKNOWLEDGEMENTS}

To Dr Paul Michels (Brussels) for giving us the antibody against Trypanosoma brucei glycerolphosphate dehydrogenase.

\section{REFERENCES}

Affranchino JL, De Tarlovsky MNS, Stoppani AOM 1985. Respiratory control in mitochondria from Trypanosoma cruzi. Mol Biochem Parasitol 16: 289298.

Bergmeyer HU 1983. Methods of Enzymatic Analysis, Academic Press, New York.

Cazzulo JJ 1994. Intermediate metabolism in Trypanosoma cruzi. J Bioenerg Biomembr 26: 157-165.
Cazzulo JJ, Arauzo S, Franke de Cazzulo BM, Cannata JJB 1988. On the production of glycerol and L-alanine during the aerobic fermentation of glucose by trypanosomatids. FEMS Microbiol Lett 51: 187-192.

Concepcion JL, Chataing B, Dubourdieu M 1999. Purification and properties of phosphoglucose isomerases of Trypanosoma cruzi. Comp Biochem Physiol 122: 211-222.

Concepcion J, Gonzalez-Pacanowska D, Urbina JA 1998. 3-hydroxy-3-methyl-glutaryl-CoA reductase in Trypanosoma cruzi: subcellular localization and kinetic properties. Arch Biochem Biophys 352: 114120.

Engel JC, Doyle PS, Dvorak JA 1990. Isolate-dependent differences in the oxidative metabolism of Try- 
panosoma cruzi epimastigotes. Mol Biochem Parasitol 39: 69-76.

Felix CR, Caldas RA, Ceron CR, Roitman L 1978. Cyanide sensitive and insensitive respiration of Trypanosoma cruzi. Ann Trop Med Parasitol 72: 89-91.

Niesel DW, Bewley GC, Lee C-Y, Armstrong FB 1982. Glycerol-3-phosphate dehydrogenase from Drosophila melanogaster. Meth Enzymol 89: 296-301.

Opperdoes FR 1987. Compartmentation of carbohydrate metabolism in trypanosomes. Ann Rev Microbiol 41: 127-151.

Sanchez-Moreno M, Fernandez-Becerra MC, CastillaCalvente JJ, Osuna A 1995. Metabolic studies by ${ }^{1} \mathrm{H}$ NMR of different forms of Trypanosoma cruzi as obtained by "in vitro" culture. FEMS Lett 133: 119-125.

Stoppani AOM, Docampo R, Boiso JF de, Frasch ACC 1980. Effect of inhibitors of electron transport and oxidative phosphorylation on Trypanosoma cruzi and growth. Mol Biochem Parasitol 2: 3-21.

Taylor MB, Guttridge E 1987. Trypanosoma cruzi subcellular distribution of glycolytic and some related enzymes of epimastigotes. Exp Parasitol 63: 84-97.

Tielens AGM, Van Hellemond JJ 1998. Differences in energy metabolism between Trypanosomatidae. Parasitol Today 14: 265-271.

Urbina JA 1994. Intermediate metabolism of Trypanosoma cruzi. Parasitol Today 10: 107-110.

Urbina JA, Machin I, Jurado L 1993. Limitations of paradigms: studies on the intermediary metabolism of Trypanosoma cruzi. Biol Res 26: 81-88.

Urbina JA, Osorno CE, Rojas A 1990. Inhibition of phosphoenolpyruvate carboxykinase from Trypanosoma (Schizotrypanum) cruzi epimastigotes by 3mercaptopicolinic acid: in vitro and in vivo studies. Arch Biochem Biophys 282: 91-99. 
T. cruzi $\alpha$-glycerophosphate Dehydrogenase • JL Concepcion et al. 